\title{
INTERSTITIAL INCISIONAL HERNIA FOLLOWING APPENDECTOMY
}

Singapore Med J 2013; 54(9): 531-532 doi:10.11622/smedj.2013177

Appendectomy is a very common surgical procedure, and post-appendectomy incisional hernia is a very rare complication that occurs in less than $0.12 \%$ of patients. ${ }^{(1,2)}$ An interstitial incisional hernia is one in which the hernia sac is located between the layers of the abdominal wall. We report a case of post-appendectomy interstitial incisional hernia diagnosed using computed tomography $(\mathrm{CT})$.

An 18-year-old man presented with a sudden onset of colicky abdominal pain and vomiting. There was no fever and the abdomen was soft with generalised tenderness. Digital rectal examination showed an empty rectum. The patient had a recent history of open appendectomy for perforated appendicitis, which was performed seven days prior to presentation. Abdominal radiography demonstrated multiple dilated small bowel loops with no intraperitoneal free air (Fig. 1). As the patient had a past history of contrast reaction, unenhanced CT of the abdomen and pelvis was performed. This showed dilated proximal small bowel loops with a transitional zone in the right lower abdomen, where a knuckle of small bowel was seen on the undersurface of the intact external oblique aponeurosis at the previous operation site. A 3-cm muscle defect was demonstrated in the internal oblique and transversus abdominis muscles, through which the hernia sac penetrated to the undersurface of the external oblique aponeurosis (Figs. 2a \& b). A diagnosis of interstitial incisional hernia was made.

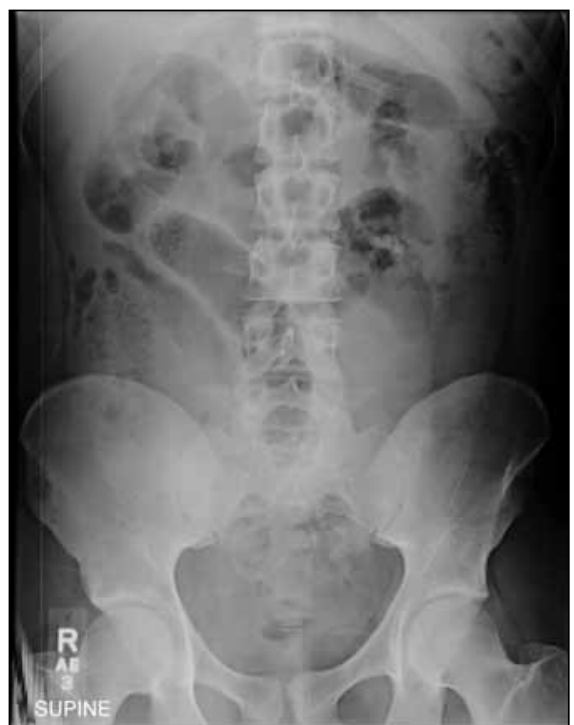

Fig. 1 Supine abdominal radiograph shows multiple dilated small bowel loops with no intraperitoneal free air.

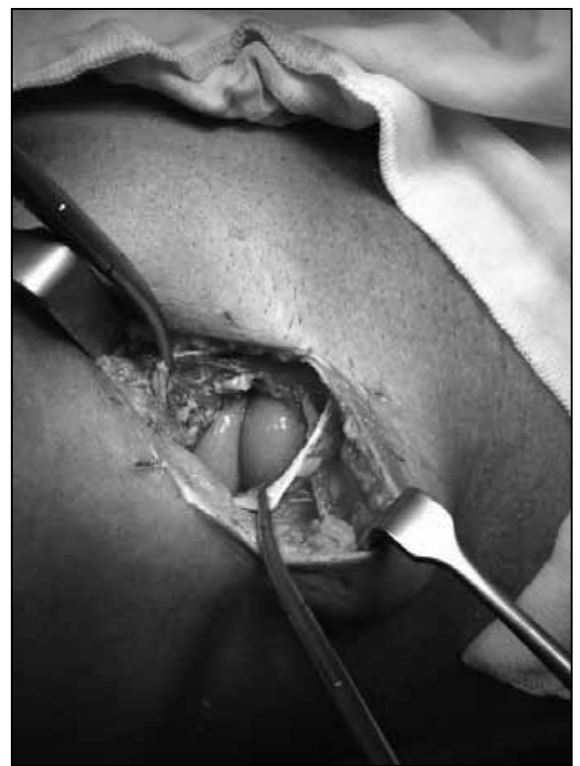

Fig. 3 Intraoperative photograph shows a healthy loop of the herniated bowel located between the muscle layers.
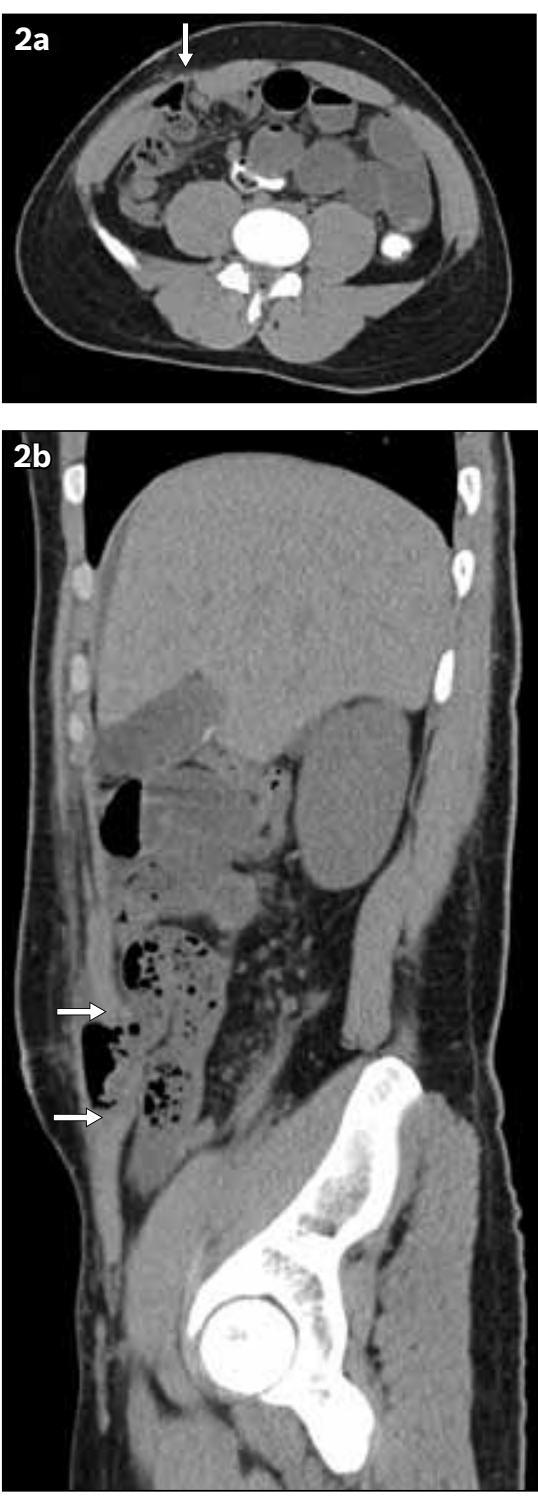

Fig. 2 Unenhanced (a) axial and (b) sagittal CT images of the abdomen and pelvis show dilated proximal small bowel loops with a transitional zone, where a knuckle of small bowel is seen on the undersurface of the intact external oblique aponeurosis at the site of the previous scar. A $3-\mathrm{cm}$ muscle defect (arrows) is seen in the internal oblique and transversus abdominis muscles. 
The patient underwent re-exploration of the appendectomy wound. The subcutaneous tissue was found to be healthy. The intact external oblique aponeurosis was opened up, and an underlying defect in the muscle layer with a knuckle of small bowel caught in the muscle defect was seen (Fig. 3). The small bowel was slightly bruised but healthy. A transition point was noted at the knuckle of small bowel, with distal bowel collapse and a slightly distended proximal bowel. The knuckle of small bowel was released and reduced into the abdominal cavity. Anatomical closure of the muscle and the external oblique aponeurosis was performed. The patient was discharged well on postoperative Day 3.

Incisional hernias usually manifest during the first few months after surgery. ${ }^{(3)}$ The occurrence of these hernias depends on a number of factors, including patient factors such as old age, male sex, obesity, smoking, diabetes mellitus and steroid use, and some surgical factors such as emergency surgery, bowel surgery, infection, and suture type and technique. ${ }^{(4)}$ In our present case, the contributing factors were male sex, emergency surgery and bowel surgery. It is possible that in our young patient, infection in the deep muscle layers leading to muscle dehiscence and subsequent hernia formation was the cause of the hernia, which occurred seven days post appendectomy.

Two types of incisional hernias can occur after appendectomy. In the more common type, the hernia passes through all layers of the abdominal wall. In the less common interstitial type, the hernia passes through a defect in the transversus abdominis and internal oblique muscles, but not through the intact aponeurosis of the external oblique muscles. ${ }^{(2)}$ This less common type of hernia can be easily missed. In patients suspected to have this condition, ultrasonography or CT helps confirm the diagnosis. CT can easily demonstrate defects in the abdominal wall muscles and common complications of hernias such as intestinal obstruction, incarceration and strangulation. ${ }^{(3)}$ In our case, CT showed a muscle defect in the internal oblique and transversus abdominis muscles through which the hernia sac penetrated to the undersurface of the intact external oblique aponeurosis. A transitional point at the site of the hernia was also seen, revealing dilated proximal loops and collapsed distal loops consistent with intestinal obstruction.

Treatment of post-appendectomy incisional hernia consists of either anatomical repair or application of a sheet of polypropylene mesh between the peritoneum and transversus abdominis muscle. ${ }^{(5)}$ Our patient underwent anatomical repair after reduction. This case assumes significance, as it involves the interstitial type of post-appendectomy incisional hernia, which should be considered as a rare but important complication post appendectomy. Early diagnosis and management of such hernias helps prevent complications.

\section{Yours sincerely,}

Ehab Shaban Mahmoud Hamouda1, Bo Chuan Tan², Wilfred CG Peh ${ }^{3}$

${ }^{1}$ Department of Diagnostic and Interventional Imaging, KK Women's and Children's Hospital, ${ }^{2}$ Department of General Surgery, ${ }^{3}$ Department of Diagnostic

Radiology, Khoo Teck Puat Hospital, Alexandra Health, Singapore. ehab.shaban.mahmoud@kkh.com.sg

\section{References}

1. Kostantakos AK, Zollinger RM Jr. Repair of McBurney incisional hernias after open appendectomy. Curr Surg 2000; 57:79-80.

2. Abrahamson J. Hernias. In: Zinner MG, Schwartz SI, Ellis H, eds. Maingot's Abdominal Operations. 10th ed. Connecticut: Appleton and Lange, 1997: 479-80.

3. Aquirre DA, Santosa AC, Casola G, Sirlin CB. Abdominal wall hernias: imaging features, complications, and diagnostic pitfalls at multi-detector row CT. Radiographics 2005; 25:1501-20.

4. Abou-Nukta F, Bakhos C, Arroyo K, et al. Effects of delaying appendectomy for acute appendicitis for 12 to 24 hours. Arch Surg 2006; 141:504-6; discussion 506-7.

5. Duce AM, Lozano O, Villeta R et al. Incisional hernia following appendectomy. Surgical experience. Hernia 1998; 2:169-71. 